\title{
Borderline Ovarian Mixed Epithelial Tumor/Atypical Proliferative Ovarian Mixed Epithelial Tumor
}

National Cancer Institute

\section{Source}

National Cancer Institute. Borderline Ovarian Mixed Epithelial Tumor/Atypical Proliferative

Ovarian Mixed Epithelial T umor. NCI Thesaurus. Code C158616.

A low grade, non-invasive mixed epithelial proliferative neoplasm that arises from the ovary. In most cases is composed of serous and endocervical-type mucinous cells. 